\title{
Disparity in the Fear of Falling Between Urban and Rural Residents in Relation With Socio-economic Variables, Health Issues, and Functional Independency
}

\author{
Hyungpil Cho, $\mathrm{MD}^{1}$, Seung Jun Seol, $\mathrm{MD}^{1}$, Do Hyun Yoon, $\mathrm{MD}^{1}$, \\ Mi Jung Kim, $\mathrm{MD}^{1}$, Bo Youl Choi, $\mathrm{MD}^{2}$, Taikon Kim, $\mathrm{MD}^{1}$
}

\begin{abstract}
${ }^{1}$ Department of Rehabilitation Medicine, Hanyang University Medical Center, Hanyang University College of Medicine, Seoul; ${ }^{2}$ Department of Preventive Medicine, Hanyang University College of Medicine, Seoul, Korea
\end{abstract}

\begin{abstract}
Objective To investigate disparities in the fear of falling between urban and rural communities in relation to socio-demographics, health status, and functional level.

Methods A total of 974 subjects aged 40 years or older participated in this study (335 urban residents and 639 rural). They completed a questionnaire about socio-demographics, health-related variables, and experience with falls. We employed both direct questioning and the Korean version of Falls Efficacy Scale-International (KFES-I) to investigate fear of falling in terms of perceptive fear and higher level of concern over falling during daily activities. The Korean version of Instrumental Activities of Daily Living was used to assess functional independency.

Results Aging, female gender, fall history, and the presence of chronic medical problems were independently associated with higher prevalence for the fear of falling. Both perceptive fear of falling and a higher level of concern over falling were more prevalent in the rural senior population compared with those in the urban population when they had the following characteristics: lower income or educational background, physical laborer or unemployed, no chronic medical morbidity, or functional independency in daily activities.

Conclusion The disparity in the fear of falling between the two areas is thought to be related to age structure, and it may also exist in healthy or functionally independent senior populations under the influence of socioenvironmental factors. A senior population with lower socio-economic status residing in a rural area might be related with a greater vulnerability to the fear of falling. We should consider regional characteristics when we design fall-related studies or develop fall-prevention programs at the community level.
\end{abstract}

Keywords Accidental falls, Geriatric assessment, Activities of daily living

Received April 23, 2013; Accepted August 28, 2013

Corresponding author: Taikon Kim

Department of Rehabilitation Medicine, Hanyang University Medical Center, Hanyang University College of Medicine, 222 Wangsimni-ro, Seongdonggu, Seoul 133-791, Korea

Tel: +82-2-2290-9349, Fax: +82-2-2282-0772, E-mail: rehman@naver.com

(c) This is an open-access article distributed under the terms of the Creative Commons Attribution Non-Commercial License (http://creativecommons.org/ licenses/by-nc/3.0) which permits unrestricted noncommercial use, distribution, and reproduction in any medium, provided the original work is properly cited. Copyright $\odot 2013$ by Korean Academy of Rehabilitation Medicine 


\section{INTRODUCTION}

Falling is a major medical problem that can cause serious injuries including fracture and brain injury, and may even lead to death. According to worldwide statistics in 2000 , approximately 283,000 people were injured seriously from falls [1]. More than $50 \%$ of seniors with a history of falls experience them again [2], and $25 \%$ appear to have restrictions in their activities of daily living (ADL) due to physical injury and fear of falling. Fear of falling is thought to be a problematic consequence of falls, and people with experience of falls feel that fear of falling is strongest compared with other fears $[3,4]$.

Prevalence for fear of falling in community senior citizens ranges from $25 \%-50 \%$ depending on the study [5-8], and is higher in females and those who have experienced any fall $[7,9,10]$. Fear of falling is thought to share risk factors with falls [11] and may have an equivalent importance to a health issue [12]. Fear of falling is related to a subjective feeling of unhealthiness and leads to restrictions in ADL, deteriorated physical functioning, limited functional independence, and damaged identity $[3,12$ 14]. Fear of falling is not only the immediate result of falls [15] but also a risk factor for falls $[7,11,12]$ which creates a vicious cycle between falls and fear of falling [11].

Many attempts have been made to assess fear of falling. Subjects have been directly asked whether they had a fear of falling, but this was inaccurate and had low predictable value. Tinetti et al. [16] defined fear of falling as "low perceived self-efficacy in avoiding falls during essential, nonhazardous activities of daily living" and developed the Falls Efficacy Scale (FES) in 1990. To complement low sensitivity to detect fear of falling for individuals with proper ADL [11,12], the Falls Efficacy Scale-International (FES-I) was developed by Yardley et al. [17] in 2005 and consists of 16 items (six additional and 10 existing items). The FES-I is a recommended tool in both research and clinical settings [18] and has been translated into various languages including Korean [19-21].

It is important to expose the relationship between fear of falling and related variables and risk factors for falls. Falls result from compound implications of both intrinsic and extrinsic risk factors [22]. Intrinsic factors are composed of physical and psychological status of the person, and the extrinsic factors are environmental issues [14]. Among extrinsic factors, regional characteristics may play an important role in the incidence of fall because they are directly related to the home and occupational environment and are indirectly associated with intrinsic factors. Fear of falling is both a consequence and risk factor for falls and shares some risk factors with falls [3,7,9,11-15]. Therefore, we hypothesized that fear of falling may be influenced by regional characteristics. Thus, investigating regional disparity of the prevalence for a fear of falling in relation with health-related and socio-environmental factors will provide a clue to understand and reduce fear of falling. This approach will contribute to establish fall prevention programs at the community level. However, most studies have been conducted only in a certain area or facility or recruited participants from regional medical centers, but few studies have investigated the disparity of fear of falling in rural and urban areas and its relationship with other fall-related risk factors [23-29].

Many researchers had suggested that fear of falling is related with impaired functional independency $[5,8,12,13,30,31]$, and that fear of falling is related with decline of Instrumental Activities Of Daily Living (IDAL) in regional studies in Korea [28,32].

In this study, we investigated the disparity for the fear of falling between urban and rural areas in relation to socio-demographics, health issues and medical morbidities, and functional level in daily activities.

\section{MATERIALS AND METHODS}

\section{Subjects}

This study was conducted as part of a 2008 Community Health Survey. Among 1,685 participants, 974 participants (335 from urban and 639 from rural community) aged 40 years or older participated.

\section{Methods}

\section{Fear of falling assessment}

This study approached fear of falling in two ways: 1 ) direct questioning focused on perceptive fear of falling and 2) the Korean version of FES-I (KFES-I) was used to measure the confidence in avoiding falling during daily activities by means of the level of concern over falling. The direct questions asked whether participants had a fear of falling. The KFES-I has 16 items, and each item can be scored from one to four (total score 16-64). According to the cut-point suggested by Delbaere et al. [18], 
Table 1. Characteristics of subjects $(n=8)$

\begin{tabular}{|c|c|c|c|c|}
\hline Characteristic & Urban $(n=335)$ & Rural (n=639) & p-value ${ }^{\text {a) }}$ & $\chi^{2}$ \\
\hline Age (yr) & $54.5 \pm 10.9$ & $61.4 \pm 11.9$ & 0.028 & \\
\hline $40-49$ & $140(41.8)$ & $130(20.3)$ & $<0.001$ & 70.865 \\
\hline $50-59$ & $83(24.8)$ & $157(24.6)$ & & \\
\hline $60-69$ & $75(22.4)$ & $160(25.0)$ & & \\
\hline$\geq 70$ & $37(11.0)$ & $192(30.1)$ & & \\
\hline \multicolumn{5}{|l|}{ Gender } \\
\hline Male & $145(43.3)$ & $294(46.0)$ & 0.416 & 0.660 \\
\hline Female & $190(56.7)$ & $345(54.0)$ & & \\
\hline \multicolumn{5}{|l|}{ Household (generations living with) } \\
\hline 2 or 3 & $253(75.5)$ & $286(44.8)$ & 84.166 & $<0.001$ \\
\hline 1 & $82(24.5)$ & $353(55.2)$ & & \\
\hline \multicolumn{5}{|l|}{ Living with spouse } \\
\hline Yes & $271(80.9)$ & $496(77.6)$ & 0.235 & 1.408 \\
\hline None & $64(19.1)$ & $143(22.4)$ & & \\
\hline \multicolumn{5}{|l|}{ Educational background (yr) } \\
\hline$\geq 10$ & $181(54.0)$ & $233(36.5)$ & 27.752 & $<0.001$ \\
\hline$\leq 9$ & $154(46.0)$ & $406(63.5)$ & & \\
\hline \multicolumn{5}{|l|}{ Occupation } \\
\hline Profession, office worker & $100(29.9)$ & $129(20.2)$ & 17.159 & $<0.001$ \\
\hline Physical laborer & $80(23.9)$ & $222(34.7)$ & & \\
\hline None & $155(46.3)$ & $288(45.1)$ & & \\
\hline \multicolumn{5}{|l|}{ Income $\left(10^{4} \mathrm{KRW}\right)$} \\
\hline$>100$ & $237(78.5)$ & $296(54.5)$ & $<0.001$ & 47.855 \\
\hline$\leq 100$ & $65(21.5)$ & $247(45.5)$ & & \\
\hline \multicolumn{5}{|l|}{ Housing type } \\
\hline Apartment or condominium & $158(47.2)$ & $37(6.0)$ & $<0.001$ & 234.955 \\
\hline Others & $177(52.8)$ & $602(94.0)$ & & \\
\hline Falls in the past $1 \mathrm{yr}(+)$ & $47(14.1)$ & $86(13.5)$ & 0.791 & 0.070 \\
\hline Chronic disease or health problem (+) & $188(56.1)$ & $398(62.3)$ & 0.062 & 3.486 \\
\hline Neurologic disorder (+) & $10(3.0)$ & $36(5.6)$ & 0.064 & 3.427 \\
\hline Musculoskeletal disorder (+) & $69(20.6)$ & $164(25.7)$ & 0.078 & 3.102 \\
\hline Cardiovascular disease $(+)$ & $92(27.5)$ & $218(34.1)$ & 0.034 & 4.484 \\
\hline Asthma (+) & $8(2.4)$ & $25(3.9)$ & 0.212 & 1.560 \\
\hline Gastrointestinal ulcer (+) & $19(5.7)$ & $17(2.7)$ & 0.018 & 5.599 \\
\hline Diabetes (+) & $32(9.6)$ & $91(14.2)$ & 0.036 & 4.379 \\
\hline Urinary incontinence $(+)$ & $23(6.9)$ & $25(3.9)$ & 0.043 & 4.091 \\
\hline Anemia (+) & $30(9.0)$ & $16(2.5)$ & $<0.001$ & 20.328 \\
\hline Cancer (+) & $11(3.3)$ & $19(3.0)$ & 0.790 & 0.071 \\
\hline Visual problem (+) & $25(7.5)$ & $41(6.4)$ & 0.537 & 0.381 \\
\hline Auditory problem (+) & $11(3.3)$ & $55(8.6)$ & 0.002 & 9.860 \\
\hline \multicolumn{5}{|l|}{ Smoking } \\
\hline Never & $194(57.9)$ & $378(59.1)$ & 0.929 & 0.147 \\
\hline Past & $68(20.3)$ & $127(19.9)$ & & \\
\hline Current & $73(21.8)$ & $134(21.0)$ & & \\
\hline
\end{tabular}


Table 1. Continued

\begin{tabular}{lcccc}
\hline \multicolumn{1}{c}{ Characteristic } & Urban $(\mathbf{n}=\mathbf{3 3 5})$ & Rural $(\mathbf{n}=\mathbf{6 3 9})$ & p-value $^{\text {a) }}$ & $\chi^{\mathbf{2}}$ \\
\hline Drinking & & & & \\
No drinking & $102(30.5)$ & $314(49.1)$ & $<0.001$ & 76.281 \\
Moderate drinking & $166(49.6)$ & $142(22.2)$ & & \\
\hline Heavy drinking & $67(20.0)$ & $183(28.6)$ & & 15.044 \\
Dependency in K-IADL & $26(7.8)$ & $107(16.7)$ & $<0.001$ & \\
\hline K-IADL score & $10.5 \pm 2.3$ & $11.0 \pm 2.9$ & 0.001 & \\
\hline
\end{tabular}

Values are presented as mean \pm standard deviation or number (\%).

KRW, Korean Won; K-IADL, Korean version of Instrumental Activities of Daily Living.

${ }^{a)} \mathrm{p}$-values were estimated by $\chi^{2}$-test or t-test, ${ }^{\text {b) }}$ regarded as dependent if dependent in at least one of the items of the K-IADL.

we regarded a score of 23 or more as having a higher level of concern over falling.

\section{Functional independency assessment}

We assessed participants' functional independency in daily activities using the Korean version of IADL (K-IADL) [33]. This questionnaire consisted of 10 items: ability to use the telephone, shopping, food preparation, housekeeping, laundry, mode of transportation, responsibility for one's own medication, ability to handle finances, grooming, and short distance outings. Participants were regarded as dependent if they showed dependency on at least one of the K-IADL items.

\section{Health-related variables}

We collected participant health-related information, including drinking and smoking habits, chronic medical problem (neurologic, musculoskeletal, cardiovascular diseases, asthma, gastrointestinal ulcer, diabetes, urinary incontinence, anemia, cancer, and visual and auditory problems), and history of falls. We classified the participants into three groups according to their drinking habits: 1) no drinking in the last year, 2) moderate drinking (once per week or less and seven cups or less at a time) and 3) heavy drinking (more than once per week or more than seven cups at a time). They were also divided into the following three categories of smoking: non-smoker, previous smoker, and current smoker. For each medical problem, two questions were asked: "I have had it in my life" and "I have been diagnosed with it by a doctor", and it was recognized only when the participants answered "Yes" to both questions.

\section{Socio-demographic variables}

Socio-demographic information was obtained using a predefined Community Health Survey questionnaire, including age, gender, occupation, marital status, family make-up (single generation vs. two or three generations living together), types of housing (apartment or condominium vs. other types), educational background, and average income. Occupation was classified into three categories of professional practice or office worker, physical laborer including farmer, and none. Educational background was divided into the following two groups: 1) up to nine years (junior high school graduate) and 2) over 10 years (high school graduate or higher). Income was answered in two ways: 1) one million Korean Won (KRW) per family or less and 2) more than one million KRW per family.

\section{Statistical analyses}

The chi-square test was used to compare all variables between urban and rural residents. The prevalence of the perceptive fear of falling and higher level of concern over falling in each subgroup according to socio-demographic or health-related variables was analyzed in the urban and rural residents, respectively, and the differences between the two regions were compared using the chi-square test. Univariate analyses with the chi-square test were conducted for each variable to evaluate the association with the prevalence of perceptive fear of falling and higher level of concern over falling. Variables with significant association went to the next step in the analysis. Binary logistic regression analyses was performed to test whether those variables were independently associated with perceptive fear of falling or higher level of concern over 
Table 2. Comparison of the prevalence of perceptive fear of falling and higher level of concern over falling between the urban and rural areas

\begin{tabular}{|c|c|c|c|c|c|c|c|c|}
\hline & \multicolumn{2}{|c|}{$\begin{array}{l}\text { Perceptive fear of } \\
\text { falling }\end{array}$} & \multirow[t]{2}{*}{ p-value } & \multirow[t]{2}{*}{$\chi^{2}$} & \multicolumn{2}{|c|}{$\begin{array}{l}\text { Higher level of con- } \\
\text { cern over falling }\end{array}$} & \multirow[t]{2}{*}{ p-value } & \multirow[t]{2}{*}{$\chi^{2}$} \\
\hline & Urban & Rural & & & Urban & Rural & & \\
\hline All participants & $107(31.9)$ & $309(48.4)$ & $<0.001$ & 24.207 & $30(9.0)$ & $130(20.7)$ & $<0.001$ & 21.594 \\
\hline \multicolumn{9}{|l|}{ Age (yr) } \\
\hline $40-49$ & $23(16.4)$ & $19(14.6)$ & 0.681 & 0.169 & $0(0.0)$ & $4(3.1)$ & 0.114 & 4.341 \\
\hline $50-59$ & $24(28.9)$ & $41(26.1)$ & 0.642 & 0.216 & $5(6.0)$ & $8(5.1)$ & 0.771 & 0.085 \\
\hline $60-69$ & $33(44.0)$ & $91(56.9)$ & 0.065 & 3.396 & $14(18.7)$ & $27(17.3)$ & 0.800 & 0.064 \\
\hline$\geq 70$ & $27(73.0)$ & $158(82.3)$ & 0.188 & 1.735 & $11(29.7)$ & $91(48.9)$ & 0.032 & 4.582 \\
\hline \multicolumn{9}{|l|}{$\begin{array}{l}\text { Household } \\
\text { (generations living with) }\end{array}$} \\
\hline 2 or 3 & $72(28.5)$ & $117(40.9)$ & 0.003 & 9.140 & $18(7.1)$ & $50(17.9)$ & $<0.001$ & 13.657 \\
\hline 1 & $35(42.7)$ & $192(54.4)$ & 0.056 & 3.656 & $12(14.6)$ & $80(23.0)$ & 0.097 & 2.754 \\
\hline \multicolumn{9}{|l|}{ Living with spouse } \\
\hline Yes & $74(27.3)$ & $223(45.0)$ & $<0.001$ & 23.017 & $12(4.4)$ & $90(18.4)$ & $<0.001$ & 29.144 \\
\hline None & $33(51.6)$ & $86(60.1)$ & 0.249 & 1.331 & $18(28.1)$ & $40(28.8)$ & 0.924 & 0.009 \\
\hline \multicolumn{9}{|l|}{ Educational background (yr) } \\
\hline$\geq 10$ & $33(18.2)$ & $59(25.3)$ & 0.085 & 2.963 & $3(1.7)$ & $10(4.3)$ & 0.128 & 2.318 \\
\hline$\leq 9$ & $74(48.1)$ & $250(61.6)$ & 0.004 & 8.376 & $27(17.5)$ & $120(30.3)$ & 0.002 & 9.234 \\
\hline \multicolumn{9}{|l|}{ Occupation } \\
\hline Profession, office worker & $17(17.0)$ & $26(20.2)$ & 0.554 & 0.368 & $1(1.0)$ & $5(3.9)$ & 0.180 & 1.795 \\
\hline Physical laborer & $20(25.0)$ & $104(46.8)$ & 0.001 & 11.598 & $3(3.8)$ & $31(14.3)$ & 0.011 & 6.400 \\
\hline None & $70(45.2)$ & $179(62.2)$ & 0.001 & 11.819 & $26(16.8)$ & $94(33.3)$ & $<0.001$ & 13.769 \\
\hline \multicolumn{9}{|l|}{ Income $\left(10^{4} \mathrm{KRW}\right)$} \\
\hline$>100$ & $60(25.3)$ & $105(35.5)$ & 0.012 & 6.352 & $12(5.1)$ & $33(11.5)$ & 0.010 & 6.712 \\
\hline$\leq 100$ & $33(50.8)$ & $143(57.9)$ & 0.303 & 1.063 & $14(21.5)$ & $69(28.3)$ & 0.276 & 1.187 \\
\hline \multicolumn{9}{|l|}{ Housing type } \\
\hline Apartment & $43(27.2)$ & $6(16.2)$ & 0.165 & 1.928 & $9(5.7)$ & $2(5.4)$ & 0.938 & 0.006 \\
\hline Others & $64(36.2)$ & $303(50.3)$ & 0.001 & 11.029 & $21(11.9)$ & $128(21.7)$ & 0.004 & 8.355 \\
\hline \multicolumn{9}{|l|}{ Fall in the past year } \\
\hline$(-)$ & $76(26.4)$ & $248(44.8)$ & $<0.001$ & 27.241 & $19(6.6)$ & $91(16.7)$ & $<0.001$ & 16.713 \\
\hline$(+)$ & $31(66.0)$ & $61(70.9)$ & 0.553 & 0.352 & $11(23.4)$ & $39(46.4)$ & 0.009 & 6.770 \\
\hline
\end{tabular}

Chronic disease or health problem

Neurologic disorder

\begin{tabular}{lrrrrrrrr}
$(-)$ & $98(30.2)$ & $281(46.6)$ & $<0.001$ & 23.642 & $23(7.1)$ & $111(18.8)$ & $<0.001$ & 22.761 \\
$(+)$ & $9(90.0)$ & $28(77.8)$ & 0.389 & 0.743 & $7(70.0)$ & $19(52.8)$ & 0.331 & 0.945 \\
$\begin{array}{l}\text { Musculoskeletal disorder } \\
(-)\end{array}$ & & & & & & & \\
$(+)$ & $62(23.3)$ & $185(38.9)$ & $<0.001$ & 18.767 & $12(4.5)$ & $60(12.8)$ & $<0.001$ & 13.134 \\
$(+)$ & $45(65.2)$ & $124(75.6)$ & 0.105 & 2.633 & $18(26.1)$ & $70(43.8)$ & 0.012 & 6.357 \\
Cardiovascular disease & & & & & & & & \\
$(-)$ & $66(27.2)$ & $168(39.9)$ & 0.001 & 10.965 & $14(5.8)$ & $72(17.3)$ & $<0.001$ & 18.114 \\
$(+)$ & $41(44.6)$ & $141(64.7)$ & 0.001 & 10.797 & $16(17.6)$ & $58(27.2)$ & 0.073 & 3.222 \\
\hline
\end{tabular}


Table 2. Continued

\begin{tabular}{|c|c|c|c|c|c|c|c|c|}
\hline & \multicolumn{2}{|c|}{$\begin{array}{c}\text { Perceptive fear of } \\
\text { falling }\end{array}$} & \multirow[t]{2}{*}{ p-value } & \multirow[t]{2}{*}{$\chi^{2}$} & \multicolumn{2}{|c|}{$\begin{array}{l}\text { Higher level of con- } \\
\text { cern over falling }\end{array}$} & \multirow[t]{2}{*}{ p-value } & \multirow[t]{2}{*}{$\chi^{2}$} \\
\hline & Urban & Rural & & & Urban & Rural & & \\
\hline \multicolumn{9}{|l|}{ Asthma } \\
\hline$(-)$ & $103(31.5)$ & $288(46.9)$ & $<0.001$ & 20.855 & $28(8.6)$ & $122(20.2)$ & $<0.001$ & 21.096 \\
\hline$(+)$ & $4(50.0)$ & $21(84.0)$ & 0.051 & 3.815 & $2(25.0)$ & $8(33.3)$ & 0.660 & 0.194 \\
\hline \multicolumn{9}{|l|}{ Gastrointestinal ulcer } \\
\hline$(-)$ & $97(30.7)$ & $299(48.1)$ & $<0.001$ & 25.931 & $27(8.6)$ & $123(20.1)$ & $<0.001$ & 20.459 \\
\hline$(+)$ & $10(52.6)$ & $10(58.8)$ & 0.709 & 0.139 & $3(15.8)$ & $7(41.2)$ & 0.090 & 2.882 \\
\hline \multicolumn{9}{|l|}{ Diabetes } \\
\hline$(-)$ & $93(30.7)$ & $253(46.2)$ & $<0.001$ & 19.366 & $24(7.9)$ & $105(19.4)$ & $<0.001$ & 19.886 \\
\hline$(+)$ & $14(43.8)$ & $56(61.5)$ & 0.080 & 3.055 & $6(19.4)$ & $25(28.4)$ & 0.323 & 0.976 \\
\hline \multicolumn{9}{|l|}{ Urinary incontinence } \\
\hline$(-)$ & $93(29.8)$ & $295(48.0)$ & $<0.001$ & 28.266 & $25(8.0)$ & $123(20.4)$ & $<0.001$ & 23.003 \\
\hline$(+)$ & $14(60.9)$ & $14(56.0)$ & 0.732 & 0.117 & $5(21.7)$ & $7(29.2)$ & 0.559 & 0.341 \\
\hline \multicolumn{9}{|l|}{ Anemia } \\
\hline$(-)$ & $92(30.2)$ & $300(48.2)$ & $<0.001$ & 27.162 & $27(8.9)$ & $127(20.8)$ & $<0.001$ & 20.462 \\
\hline$(+)$ & $15(50.0)$ & $9(56.3)$ & 0.686 & 0.163 & $3(10.0)$ & $3(18.8)$ & 0.401 & 0.704 \\
\hline \multicolumn{9}{|l|}{ Cancer } \\
\hline$(-)$ & $102(31.5)$ & $296(47.7)$ & $<0.001$ & 23.073 & $30(9.3)$ & $126(20.7)$ & $<0.001$ & 19.598 \\
\hline$(+)$ & $5(45.5)$ & $13(68.4)$ & 0.261 & 1.531 & $0(0.0)$ & $4(22.2)$ & 0.268 & 2.836 \\
\hline \multicolumn{9}{|l|}{ Visual problem } \\
\hline$(-)$ & $90(29.0)$ & $280(46.8)$ & $<0.001$ & 26.763 & $23(7.4)$ & $110(18.7)$ & $<0.001$ & 20.266 \\
\hline$(+)$ & $17(68.0)$ & $29(70.7)$ & 0.815 & 0.055 & $7(28.0)$ & $20(51.3)$ & 0.066 & 3.386 \\
\hline \multicolumn{9}{|l|}{ Auditory problem } \\
\hline$(-)$ & $100(30.9)$ & $270(46.2)$ & $<0.001$ & 20.386 & $26(8.0)$ & $104(18.1)$ & $<0.001$ & 16.910 \\
\hline$(+)$ & $7(63.6)$ & $39(70.9)$ & 0.632 & 0.230 & $4(36.4)$ & $26(48.1)$ & 0.475 & 0.511 \\
\hline \multicolumn{9}{|l|}{ Smoking } \\
\hline Never & $69(35.6)$ & $204(54.0)$ & $<0.001$ & 17.400 & $17(8.8)$ & $87(23.5)$ & $<0.001$ & 18.212 \\
\hline Past & $16(23.5)$ & $57(44.9)$ & 0.003 & 8.621 & $8(11.8)$ & $21(16.7)$ & 0.361 & 0.835 \\
\hline Current & $22(30.1)$ & $48(35.8)$ & 0.409 & 0.682 & $5(6.8)$ & $22(16.7)$ & 0.047 & 3.961 \\
\hline \multicolumn{9}{|l|}{ Drinking } \\
\hline No drinking & $50(49.0)$ & $184(58.6)$ & 0.090 & 2.871 & $19(18.6)$ & $93(30.5)$ & 0.020 & 5.394 \\
\hline Moderate drinking & $47(28.3)$ & $58(40.8)$ & 0.021 & 5.349 & $9(5.5)$ & $15(10.6)$ & 0.096 & 2.764 \\
\hline Heavy drinking & $10(14.9)$ & $67(36.6)$ & 0.001 & 10.822 & $2(3.0)$ & $22(9.7)$ & 0.030 & 4.704 \\
\hline \multicolumn{9}{|l|}{$\mathrm{IADL}^{\mathrm{c})}$} \\
\hline Independent & $94(30.4)$ & $264(49.6)$ & $<0.001$ & 29.484 & $28(9.1)$ & $113(21.6)$ & $<0.001$ & 21.551 \\
\hline Dependent & $13(50.0)$ & $45(42.1)$ & 0.464 & 0.537 & $2(7.7)$ & $17(16.2)$ & 0.271 & 1.214 \\
\hline
\end{tabular}

Values are presented as number (\%).

KRW, Korean Won; IADL, Instrumental Activities of Daily Living.

p-values were estimated by $\chi^{2}$-test.

${ }^{\text {a) }}$ Asked by direct question, ${ }^{\text {b }}$ measured by Korean version of the Falls Efficacy Scale-International, ${ }^{\text {c) }}$ regarded as dependent if dependent in at least one of the items of K-IADL. 
falling. We also divided the participants into four groups according to age (40-59 years vs. 60 years or older) and region (urban vs. rural), and performed the MannWhitney U-test to check for differences in the KFES-I scores among the groups. A p-value $<0.05$ was considered significant in all analyses. Data analyses were performed using the SPSS program for Windows, ver. 18.0 (SPSS Inc., Chicago, IL, USA).

\section{RESULTS}

\section{General characteristics}

The senior population occupied a larger proportion in the rural than the urban participants, and the mean age of the rural group was older than that of the urban group (Table 1). Differences in socio-demographic and healthrelated variables between areas are shown in Table 1. The incidence of falls during the past year was not significantly different between the urban and rural populations. Females showed a higher incidence of falls than males in both the urban $(19.0 \%$ of females and $7.6 \%$ of males, $\mathrm{p}=0.003)$ and rural areas $(16.8 \%$ of females and $9.5 \%$ of males, $\mathrm{p}=0.007)$. Dependency of IADL was more prevalent in the rural population compared with the urban. However, the K-IADL score was only slightly different between the two populations (Table 1).

\section{Prevalence of perceptive fear of falling Perceptive fear offalling}

The prevalence of perceptive fear of falling was significantly higher in the rural population than that in the urban one and increased with aging in both urban and rural areas (Table 2). However, no significant differences were observed between the area groups when we analyzed each age group separately. The prevalence of fear of falling according to other socio-demographic and healthrelated variables is described in Table 2 .

\section{Level of concern over falling}

The rural participants showed a higher KFES-I score

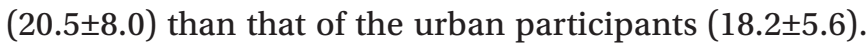
The median KFES-I scores $(5 \%, 95 \%)$ in the urban and the rural groups were $16(16,28)$ and $17(26,39)$, respectively. The prevalence of a higher level of concern was

Table 3. Variables associated with the prevalence of perceptive fear of falling and higher level of concern over falling according to univariate analyses

\begin{tabular}{|c|c|c|c|c|c|c|}
\hline & \multicolumn{3}{|c|}{ Perceptive fear of falling ${ }^{a)}$} & \multicolumn{3}{|c|}{ Higher level of concern over falling ${ }^{\text {b) }}$} \\
\hline & OR & 95\% CI & p-value & OR & 95\% CI & p-value \\
\hline \multicolumn{7}{|l|}{ Age (yr) } \\
\hline $50-59$ & 2.02 & $1.31-3.12$ & 0.002 & 3.81 & $1.23-11.85$ & 0.021 \\
\hline $60-69$ & 6.06 & $4.00-9.20$ & $<0.001$ & 14.30 & $5.04-40.59$ & $<0.001$ \\
\hline$\geq 70$ & 22.83 & $14.34-36.34$ & $<0.001$ & 55.85 & $20.10-155.17$ & $<0.001$ \\
\hline Female & 2.12 & $1.63-2.75$ & $<0.001$ & 2.02 & $1.41-2.90$ & $<0.001$ \\
\hline Rural area & 2.00 & $1.51-2.63$ & $<0.001$ & 2.65 & $1.74-4.03$ & $<0.001$ \\
\hline Household, 1 generation & 2.02 & $1.56-2.62$ & $<0.001$ & 1.86 & $1.32-2.62$ & $<0.001$ \\
\hline Not living with spouse & 2.14 & $1.57-2.92$ & $<0.001$ & 2.58 & $1.78-3.73$ & $<0.001$ \\
\hline Educational background, $\leq 9 \mathrm{yr}$ & 4.81 & $3.61-6.40$ & $<0.001$ & 11.20 & $6.24-20.07$ & $<0.001$ \\
\hline \multicolumn{7}{|l|}{ Occupation, } \\
\hline Physical laborer & 3.01 & $2.01-4.51$ & $<0.001$ & 4.78 & $1.97-11.60$ & 0.001 \\
\hline None & 5.55 & $3.79-8.13$ & $<0.001$ & 14.01 & $6.06-32.37$ & $<0.001$ \\
\hline Income, $\leq 1,000,000 \mathrm{KRW}$ & 2.89 & $2.12-3.86$ & $<0.001$ & 3.91 & $2.63-5.81$ & $<0.001$ \\
\hline Housing type, others & 2.65 & $1.87-3.78$ & $<0.001$ & 4.01 & $2.12-7.55$ & $<0.001$ \\
\hline Fall in the past year & 3.58 & $2.41-5.31$ & $<0.001$ & 4.05 & $2.70-6.07$ & $<0.001$ \\
\hline Chronic disease or health problems & 4.73 & $3.53-6.33$ & $<0.001$ & 6.69 & $4.02-11.14$ & $<0.001$ \\
\hline
\end{tabular}

Values were obtained by chi-square test.

OR, odds ratio; CI, confidence interval, KRW, Korean Won.

${ }^{\text {a) }}$ Asked by direct question, ${ }^{\text {b) }}$ measured by Korean version of Falls Efficacy Scale-International. 
higher in rural than in urban participants and increased with aging both in the urban and the rural populations (Table 2). When we analyzed each age group separately, a higher level of concern was more prevalent in the urban compared with the rural participants 70 years or older, but other age groups did not show any regional differences. Table 2 shows the prevalence of a higher level of concern over falling according to other variables.

Table 4. Logistic regression results for predicting perceptive fear of falling and higher level of concern over falling

\begin{tabular}{|c|c|c|c|c|c|c|c|}
\hline & No. (\%) & $\beta$ Coefficient & SE $\beta$ & Wald statistic & p-value & OR & 95\% CI \\
\hline \multicolumn{8}{|l|}{ Perceptive fear of falling ${ }^{a}$} \\
\hline \multicolumn{8}{|l|}{ Age (yr) } \\
\hline $50-59$ & $65(27.1)$ & 0.38 & 0.26 & 2.13 & 0.145 & 1.46 & $0.88-2.43$ \\
\hline $60-69$ & $124(52.8)$ & 1.14 & 0.29 & 15.41 & $<0.001$ & 3.13 & $1.77-5.54$ \\
\hline$\geq 70$ & $185(80.8)$ & 2.42 & 0.35 & 47.83 & $<0.001$ & 11.22 & $5.66-22.26$ \\
\hline Female & $272(50.8)$ & 0.88 & 0.19 & 22.00 & $<0.001$ & 2.40 & $1.67-3.47$ \\
\hline Rural area & $309(48.4)$ & 0.28 & 0.21 & 1.82 & 0.177 & 1.33 & $0.88-2.00$ \\
\hline Household, 1 generation & $227(52.2)$ & -0.18 & 0.20 & 0.81 & 0.370 & 0.84 & $0.56-1.24$ \\
\hline Not living with spouse & $119(57.5)$ & -0.3 & 0.23 & 0.02 & 0.900 & 0.97 & $0.63-1.51$ \\
\hline Educational background, $\leq 9 \mathrm{yr}$ & $324(57.9)$ & 0.21 & 0.20 & 1.03 & 0.303 & 1.23 & $0.83-1.83$ \\
\hline \multicolumn{8}{|l|}{ Occupation } \\
\hline Physical laborer & $124(41.1)$ & 0.26 & 0.27 & 0.94 & 0.331 & 1.29 & $0.77-2.17$ \\
\hline None & $249(56.2)$ & 0.10 & 0.26 & 0.14 & 0.712 & 1.10 & $0.66-1.86$ \\
\hline Income, $\leq 1,000,000 \mathrm{KRW}$ & $176(56.4)$ & 0.07 & 0.21 & 0.11 & 0.746 & 1.07 & $0.71-1.61$ \\
\hline Housing type, others & $367(47.1)$ & 0.74 & 0.27 & 0.08 & 0.781 & 1.08 & $0.64-1.82$ \\
\hline Fall in the past year & $92(69.2)$ & 0.92 & 0.25 & 13.19 & $<0.001$ & 2.498 & $1.52-4.09$ \\
\hline Chronic disease or health problems & $332(56.7)$ & 0.79 & 0.19 & 17.59 & $<0.001$ & 2.20 & $1.52-3.18$ \\
\hline \multicolumn{8}{|l|}{ Higher level of concern over falling ${ }^{\text {b) }}$} \\
\hline \multicolumn{8}{|l|}{ Age (yr) } \\
\hline $50-59$ & $13(5.4)$ & 0.28 & 0.64 & 0.19 & 0.661 & 1.33 & $0.38-4.69$ \\
\hline $60-69$ & $41(17.7)$ & 1.45 & 0.60 & 5.73 & 0.017 & 4.25 & $1.30-13.90$ \\
\hline$\geq 70$ & $102(45.7)$ & 2.34 & 0.63 & 13.99 & $<0.001$ & 10.39 & $3.05-35.41$ \\
\hline Female & $110(20.8)$ & 0.53 & 0.26 & 4.09 & 0.043 & 1.70 & $1.02-2.83$ \\
\hline Rural area & $130(20.7)$ & 0.70 & 0.31 & 5.16 & 0.023 & 2.00 & $1.10-3.65$ \\
\hline Household, 1 generation & $92(21.4)$ & -0.30 & 0.29 & 1.07 & 0.300 & 0.74 & $0.42-1.30$ \\
\hline Not living with spouse & $58(28.6)$ & 0.15 & 0.28 & 0.28 & 0.597 & 1.16 & $0.67-2.00$ \\
\hline Educational background, $\leq 9 \mathrm{yr}$ & $147(26.7)$ & 0.87 & 0.36 & 5.97 & 0.015 & 2.38 & $1.19-4.79$ \\
\hline \multicolumn{8}{|l|}{ Occupation } \\
\hline Physical laborer & $34(11.4)$ & 0.56 & 0.67 & 0.69 & 0.406 & 1.75 & $0.47-6.56$ \\
\hline None & $120(27.5)$ & 1.24 & 0.65 & 3.61 & 0.057 & 3.47 & $0.96-12.49$ \\
\hline Income, $\leq 1,000,000 \mathrm{KRW}$ & $83(26.9)$ & 0.14 & 0.29 & 0.22 & 0.643 & 1.15 & $0.65^{-2.03}$ \\
\hline Housing type, others & $149(19.4)$ & -0.05 & 0.45 & 0.01 & 0.904 & 0.95 & $0.39-2.29$ \\
\hline Fall in the past year & $50(38.2)$ & 0.92 & 2.28 & 10.91 & 0.001 & 2.50 & $1.45-4.30$ \\
\hline Chronic disease or health problems & $142(24.7)$ & 0.92 & 0.33 & 7.65 & 0.006 & 2.50 & $1.31-4.78$ \\
\hline
\end{tabular}

SE $\beta$, standard error of $\beta$ coefficient; OR, odds ratio; CI, confidence interval; KRW, Korean Won.

${ }^{\text {a) }}$ Asked by direct question $\left(\mathrm{R}^{2}=0.37, \mathrm{p}<0.001\right)$, ${ }^{\text {b) }}$ measured by Korean version of the Falls Efficacy Scale-International $\left(\mathrm{R}^{2}=0.35, \mathrm{p}<0.001\right)$. 
Variables associated with perceptive fear of falling and higher level of concern over falling

In univariate analyses, age, gender, area, household, spouse, educational background, occupation, income, housing type, fall in the past year, presence of chronic medical problems, and smoking and drinking habits

Table 5. Comparison of Falls Efficacy Scale-International scores between areas according to health related variables

\begin{tabular}{|c|c|c|c|c|c|c|}
\hline \multirow{2}{*}{ Variable } & \multicolumn{3}{|c|}{$40-59$ yr } & \multicolumn{3}{|c|}{$\geq 60 \mathrm{yr}$} \\
\hline & Urban & Rural & p-value ${ }^{\text {a) }}$ & Urban & Rural & p-value ${ }^{\text {a) }}$ \\
\hline \multicolumn{7}{|c|}{ Fall in the past year } \\
\hline No & $16.6 \pm 2.3$ & $16.8 \pm 2.1$ & 0.172 & $20.0 \pm 7.4$ & $22.4 \pm 9.0$ & 0.002 \\
\hline Yes & $17.7 \pm 3.9$ & $20.9 \pm 8.3$ & 0.384 & $24.4 \pm 9.9$ & $27.3 \pm 11.4$ & 0.276 \\
\hline \multicolumn{7}{|c|}{ Neurologic disorder } \\
\hline No & $16.6 \pm 1.8$ & $17.1 \pm 3.1$ & 0.139 & $19.7 \pm 5.5$ & $22.7 \pm 9.0$ & $<0.001$ \\
\hline Yes & NA & NA & NA & $37.0 \pm 16.0$ & $28.2 \pm 13.0$ & 0.082 \\
\hline \multicolumn{7}{|c|}{ Musculoskeletal disorder } \\
\hline No & $16.6 \pm 2.4$ & $16.7 \pm 2.5$ & 0.313 & $19.5 \pm 7.3$ & $22.0 \pm 9.3$ & 0.002 \\
\hline Yes & $17.8 \pm 2.7$ & $20.0 \pm 5.8$ & 0.239 & $23.5 \pm 9.1$ & $25.6 \pm 9.8$ & 0.112 \\
\hline \multicolumn{7}{|c|}{ Cardiovascular disease } \\
\hline No & $16.6 \pm 1.8$ & $17.0 \pm 3.2$ & 0.290 & $21.2 \pm 8.8$ & $23.1 \pm 9.3$ & 0.034 \\
\hline Yes & $17.5 \pm 4.8$ & $17.8 \pm 3.5$ & 0.471 & $21.1 \pm 7.9$ & $23.5 \pm 9.9$ & 0.039 \\
\hline \multicolumn{7}{|l|}{ Asthma } \\
\hline No & $16.7 \pm 2.5$ & $17.1 \pm 3.2$ & 0.237 & $20.9 \pm 8.2$ & $23.4 \pm 9.8$ & 0.002 \\
\hline Yes & NA & NA & NA & $24.4 \pm 9.4$ & $22.1 \pm 6.7$ & 0.659 \\
\hline \multicolumn{7}{|c|}{ Gastrointestinal ulcer } \\
\hline No & $16.7 \pm 2.5$ & $17.1 \pm 3.3$ & 0.110 & $21.1 \pm 8.4$ & $23.2 \pm 9.6$ & 0.006 \\
\hline Yes & $17.0 \pm 2.1$ & $16.4 \pm 0.9$ & 0.662 & $21.0 \pm 6.0$ & $25.6 \pm 8.8$ & 0.291 \\
\hline \multicolumn{7}{|l|}{ Diabetes } \\
\hline No & $16.7 \pm 2.5$ & $17.1 \pm 3.2$ & 0.074 & $21.2 \pm 8.8$ & $23.0 \pm 9.3$ & 0.004 \\
\hline Yes & $17.0 \pm 2.0$ & $17.0 \pm 3.4$ & 0.403 & $20.8 \pm 4.4$ & $24.6 \pm 10.6$ & 0.480 \\
\hline \multicolumn{7}{|c|}{ Urinary incontinence } \\
\hline No & $16.6 \pm 2.5$ & $17.0 \pm 3.1$ & 0.103 & $20.7 \pm 8.0$ & $23.1 \pm 9.4$ & 0.001 \\
\hline Yes & $17.7 \pm 2.5$ & $19.1 \pm 4.9$ & 0.762 & $25.8 \pm 10.2$ & $27.8 \pm 14.8$ & 1.000 \\
\hline \multicolumn{7}{|l|}{ Anemia } \\
\hline No & $16.7 \pm 2.6$ & $17.0 \pm 3.1$ & 0.123 & $21.0 \pm 8.4$ & $23.3 \pm 9.6$ & 0.002 \\
\hline Yes & $16.7 \pm 1.1$ & $20.0 \pm 6.0$ & 0.146 & $21.8 \pm 7.1$ & $21.0 \pm 9.4$ & 0.425 \\
\hline \multicolumn{7}{|l|}{ Cancer } \\
\hline No & $16.7 \pm 2.5$ & $17.1 \pm 3.3$ & 0.111 & $21.3 \pm 8.5$ & $23.3 \pm 9.7$ & 0.008 \\
\hline Yes & NA & NA & NA & $18.0 \pm 2.6$ & $21.8 \pm 7.2$ & 0.285 \\
\hline \multicolumn{7}{|l|}{ Visual problem } \\
\hline No & $16.7 \pm 2.5$ & $17.1 \pm 3.3$ & 0.112 & $20.5 \pm 8.2$ & $22.6 \pm 8.8$ & 0.001 \\
\hline Yes & $17.1 \pm 2.0$ & $17.0 \pm 1.7$ & 0.787 & $25.5 \pm 7.7$ & $30.0 \pm 14.0$ & 0.510 \\
\hline \multicolumn{7}{|l|}{ Auditory problem } \\
\hline No & $16.7 \pm 2.5$ & $17.1 \pm 3.3$ & 0.130 & $20.8 \pm 8.2$ & $22.4 \pm 8.6$ & 0.008 \\
\hline Yes & NA & NA & NA & $24.4 \pm 7.3$ & $28.5 \pm 13.6$ & 0.666 \\
\hline
\end{tabular}

Values are presented as mean \pm standard deviation.

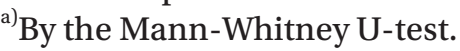


were associated with perceptive fear of falling and a higher level of concern over falling (Table 3). Binary logistic regression result with those variables showed that 60 years or older, female gender, experience of falls in the past year, and the presence of chronic medical problems was independently associated with perceptive fear of falling. Factors associated with a higher level of concern over falling were 60 years or older, female gender, living in the rural area, educational background of 9 years or less, experience of falls in the past year, and the presence

Table 6. Comparison of Falls Efficacy Scale-International scores between areas according to functional dependency

\begin{tabular}{|c|c|c|c|c|c|c|}
\hline \multirow{2}{*}{ K-IADL item } & \multicolumn{3}{|c|}{$40-59$ yr } & \multicolumn{3}{|c|}{$\geq 60 \mathrm{yr}$} \\
\hline & Urban & Rural & p-value ${ }^{a)}$ & Urban & Rural & p-value ${ }^{a)}$ \\
\hline \multicolumn{7}{|l|}{ Overall $^{\mathrm{b})}$} \\
\hline Independent & $16.7 \pm 2.5$ & $17.1 \pm 3.3$ & 0.316 & $21.2 \pm 8.5$ & $23.5 \pm 9.9$ & 0.004 \\
\hline Dependent & $16.7 \pm 1.6$ & $17.1 \pm 2.8$ & 0.516 & $20.3 \pm 3.7$ & $22.2 \pm 7.8$ & 0.755 \\
\hline \multicolumn{7}{|l|}{ 1. Grooming } \\
\hline Independent & $16.6 \pm 1.9$ & $17.6 \pm 3.3$ & 0.170 & $20.8 \pm 6.6$ & $22.9 \pm 8.1$ & 0.004 \\
\hline Dependent & NA & NA & NA & $45.8 \pm 20.4$ & $46.7 \pm 11.2$ & 0.383 \\
\hline \multicolumn{7}{|l|}{ 2. Housekeeping } \\
\hline Independent & $16.6 \pm 1.9$ & $17.0 \pm 2.4$ & 0.195 & $20.0 \pm 5.3$ & $21.9 \pm 6.8$ & 0.003 \\
\hline Dependent & $29.5 \pm 17.7$ & $23.7 \pm 10.4$ & 0.403 & $39.3 \pm 15.6$ & $35.8 \pm 14.2$ & 0.512 \\
\hline \multicolumn{7}{|l|}{ 3. Food preparation } \\
\hline Independent & $16.6 \pm 1.9$ & $17.0 \pm 2.4$ & 0.164 & $20.0 \pm 5.3$ & $22.1 \pm 7.1$ & 0.002 \\
\hline Dependent & $42.0 \pm 0.0$ & $20.6 \pm 8.7$ & 0.682 & $39.3 \pm 15.6$ & $33.3 \pm 18.1$ & 0.416 \\
\hline \multicolumn{7}{|l|}{ 4. Laundry } \\
\hline Independent & $16.6 \pm 1.9$ & $17.0 \pm 2.5$ & 0.127 & $20.0 \pm 5.4$ & $22.2 \pm 7.3$ & 0.003 \\
\hline Dependent & $18.5 \pm 7.8$ & $18.1 \pm 6.1$ & 0.752 & $34.2 \pm 16.3$ & $29.6 \pm 13.6$ & 0.551 \\
\hline \multicolumn{7}{|l|}{ 5. Going out short distance } \\
\hline Independent & $16.7 \pm 2.6$ & $17.1 \pm 3.1$ & 0.153 & $20.2 \pm 5.7$ & $22.3 \pm 7.6$ & 0.006 \\
\hline Dependent & NA & NA & NA & $42.3 \pm 15.4$ & $39.5 \pm 13.1$ & 0.241 \\
\hline \multicolumn{7}{|c|}{ 6. Using public transportation } \\
\hline Independent & $16.6 \pm 1.9$ & $17.0 \pm 2.6$ & 0.162 & $19.8 \pm 5.1$ & $21.9 \pm 7.1$ & 0.003 \\
\hline Dependent & $29.5 \pm 17.7$ & $31.3 \pm 14.2$ & 0.443 & $41.1 \pm 13.6$ & $33.6 \pm 13.6$ & 0.769 \\
\hline \multicolumn{7}{|l|}{ 7. Shopping } \\
\hline Independent & $16.6 \pm 1.9$ & $17.0 \pm 2.6$ & 0.189 & $19.8 \pm 5.0$ & $22.0 \pm 7.1$ & 0.003 \\
\hline Dependent & $42.0 \pm 0.0$ & $39.0 \pm 7.1$ & 0.353 & $40.7 \pm 14.4$ & $35.2 \pm 13.9$ & 0.679 \\
\hline \multicolumn{7}{|l|}{ 8. Handling finance } \\
\hline Independent & $16.6 \pm 1.9$ & $17.5 \pm 3.2$ & 0.228 & $21.2 \pm 7.7$ & $22.0 \pm 7.1$ & 0.004 \\
\hline Dependent & $18.6 \pm 8.2$ & $18.5 \pm 4.5$ & 0.759 & $25.0 \pm 13.5$ & $31.3 \pm 13.4$ & 0.872 \\
\hline \multicolumn{7}{|l|}{ 9. Using the telephone } \\
\hline Independent & $16.6 \pm 1.9$ & $17.2 \pm 3.3$ & 0.187 & $21.3 \pm 8.0$ & $22.6 \pm 7.9$ & 0.005 \\
\hline Dependent & NA & NA & NA & $40.0 \pm 20.0$ & $33.6 \pm 14.2$ & 0.293 \\
\hline \multicolumn{7}{|c|}{ 10. Responsibility for own medication } \\
\hline Independent & $16.7 \pm 2.6$ & $17.2 \pm 3.3$ & 0.149 & $21.5 \pm 8.2$ & $23.2 \pm 8.7$ & 0.004 \\
\hline Dependent & NA & NA & NA & $60.0 \pm 0.0$ & $41.0 \pm 14.4$ & 0.468 \\
\hline
\end{tabular}

Values are presented as mean \pm standard deviation.

K-IADL, Korean version of Instrumental Activities of Daily Living; NA, not applicable.

${ }^{a)}$ By Mann-Whitney U-test, ${ }^{\text {b) }}$ regarded as dependent if dependent in at least one of the items of K-IADL. 
of chronic medical problems (Table 4).

Comparison of KFES-I score between areas according to health-related variables and functional dependency

The KFES-I score for the rural population was higher than that of the urban population in participants age 60 years or older $(21.1 \pm 8.2$ in urban and $23.3 \pm 9.6$ in rural, $\mathrm{p}=0.003$ ), but no differences were observed according to area for participants aged $40-59$ years $(16.7 \pm 2.5$ in urban and $17.1 \pm 3.3$ in rural, $\mathrm{p}=0.163$ ). Rural residents 60 years or older showed higher KFES-I scores compared with those of urban residents when they had a chronic medical problem, but no significant differences were observed between the urban and the rural areas in the younger population ( $<60$ years) (Table 5 ). We also divided participants into pairs of groups according to the dependency of each K-IADL item and compared KFES-I scores between the two areas. In the older group ( $\geq 60$ years), rural residents showed higher KFES-I scores than urban participants independent of the K-IADL items. In contrast, no disparity in the KFES-I score was observed by region in the younger participants ( $<60$ years) (Table 6 ).

\section{DISCUSSION}

We evaluated disparities in the fear of falling, functional level, and socio-demographic and health-related variables between urban and rural communities. Experience with falls in the past year was not significantly different according to area ( $14.1 \%$ in urban and $13.5 \%$ in rural). No significant difference was observed between areas $(24.1 \%$ in urban and $17.9 \%$ in rural) in participants aged 60 years or older $(\mathrm{p}=0.170)$. These results show a lower prevalence of fall compared with some studies $(31.8 \%-51.8 \%)$ [23-26] but are comparable with other study results $(21.0 \%$ and $26.1 \%)[27,28]$. This discrepancy may be due to the inconsistency of age and gender and lack of adjustment for environmental factors. Females appeared to experience falls more frequently than males both in the urban and rural areas, which confirmed previous reports [6,9-11].

Both perceptive fear of falling and a higher level of concern over falling had higher prevalence in the rural area compared with that in the urban area and showed increased prevalence with age in both areas. However, the rural group had a larger senior population and a higher mean age compared with the urban group and there were disparities in other socio-demographic and healthrelated status between the two areas. According to the logistic regression to reveal the individual effects of variables on fear of falling, 60 years or older, female gender, experience with falls in the past year, and the presence of chronic medical problems were predictors for a higher prevalence of perceptive fear of falling and a higher level of concern over falling. In addition, rural area and lower educational background were associated with a higher level of concern over falling. Age 70 years or older was the strongest predictor for both perceptive fear of falling and a higher level of concern. Fear of falling increased with age in previous studies $[9,11,34]$, and it is the possible explanation for the regional disparity of fear of falling that may be related with disparity of age structure between the areas. This explanation was indirectly supported by the result that no regional difference was observed in the prevalence of fear of falling when we analyzed each age group separately.

We also compared KFES-I score and the prevalence of perceptive fear of falling and higher level of concern over falling between the urban and the rural participants after stratifying participants according to each variable. Participants with experience of falls in the past year or chronic medical problems did not have disparate fear of falling or KFES-I score between the areas, and there no differences in KFES-I scores between the areas in participants with dependency shown in K-IADL. Fear of falling is related with lower perceived health, medical morbidities including neurologic, cardiovascular, musculoskeletal, and visual problem, and a declined functional level [6,8-13]. Some intrinsic risk factors including experience of falls, presence of chronic medical problems, or functional dependency might have a stronger influence on the fear of falling compared with the influence of other variables related to regional characteristics.

In participants without those intrinsic risk factors (experience of fall in the past year, chronic medical problems, or functional dependency), perceptive fear of falling and a higher level of concern over falling were more prevalent in the rural area and the KFES-I score of the rural senior group was lower compared with that of urban seniors. We hypothesize that other factors contribute to the disparity of fear of falling between areas in these groups when the influence of intrinsic risk factors disappear. It may be connected with a previous report that $41 \%$ 
of falls are related to environmental factors and only $13 \%$ are caused by weakness or poor balance [29].

Among socio-demographic variables, the prevalence of perceptive fear of falling and higher level of concern over falling were different between the two areas in participants with the following characteristics: lower educational background ( $\leq 9$ years), physical laborer or no occupation, lower income $(\leq 1,000,000 \mathrm{KRW})$, and not living in an apartment or condominium. This result suggests that the rural senior population is more vulnerable to fear of falling compared with urban seniors when they are in a lower socio-economic status. Disparity of perceptive fear of falling and higher level of concern over falling between areas was also seen in participants living 2-3 generations together or living with a spouse. Although we could not find any possible explanation, we assumed that insufficient familial support in the senior population may have greater impact on fear of falling compared with regional characteristics. The relationships between individual socio-economic factors and fear of falling are still unclear, although a few researchers had reported that living alone and lower income are associated with fear of falling [8,35-37]. Additional studies will be required to investigate the effect of socio-economic status on fear of falling in relation to regional difference.

This is the first study to investigate disparities of fear of falling between urban and rural areas in relation to socio-demographics, chronic medical problems, and functional level. The results suggest that we should consider regional characteristics when we design fall-related studies or develop fall-prevention programs at the community level. However, this study had several limitations. We were unable to confirm the causal relationships as this was a cross-sectional study or explain the reason why regional disparities appeared only in the population with low socio-economic status or those without medical morbidity or functional dependency. A prospective study will be required to investigate mechanisms for our result and the cause-and-effect relationships. Sociodemographic and health related variables were asked by predefined questionnaire for a regional epidemiologic cohort study and may not have been optimized for this study. In this light, alteration or adjustment of each variable and its items should be considered in a future study. This study also had a limitation as we did not investigate the experience of falls longer than 1 year because of pos- sible recall error.

In conclusion, age, experience with fall, and health status were associated with the prevalence of fear of falling, and the regional disparity may be attributed to differences in age structure between areas. This regional disparity may exist in the healthy or functionally independent senior population under the influence of socioenvironmental factors. The senior population with lower socio-economic status residing in rural area might be related with greater vulnerability to fear of falling. Further prospective study is warranted to clarify the association between socio-environmental factors and fear of falling in terms of regional disparity and its causal relationship.

\section{CONFLICT OF INTEREST}

No potential conflict of interest relevant to this article was reported.

\section{REFERENCES}

1. Mitchell R, Curtis K, Watson WL, Nau T. Age differences in fall-related injury hospitalisations and trauma presentations. Australas J Ageing 2010;29:117-25.

2. McClure R, Turner C, Peel N, Spinks A, Eakin E, Hughes K. Population-based interventions for the prevention of fall-related injuries in older people. Cochrane Database Syst Rev 2005;(1):CD004441.

3. Howland J, Peterson EW, Levin WC, Fried L, Pordon D, Bak S. Fear of falling among the community-dwelling elderly. J Aging Health 1993;5:229-43.

4. Walker JE, Howland J. Falls and fear of falling among elderly persons living in the community: occupational therapy interventions. Am J Occup Ther 1991;45:119-22.

5. Wilson MM, Miller DK, Andresen EM, Malmstrom TK, Miller JP, Wolinsky FD. Fear of falling and related activity restriction among middle-aged African Americans. J Gerontol A Biol Sci Med Sci 2005;60:355-60.

6. Zijlstra GA, van Haastregt JC, van Eijk JT, van Rossum E, Stalenhoef PA, Kempen GI. Prevalence and correlates of fear of falling, and associated avoidance of activity in the general population of community-living older people. Age Ageing 2007;36:304-9.

7. Tinetti ME, Mendes de Leon CF, Doucette JT, Baker DI. Fear of falling and fall-related efficacy in relationship to functioning among community-living elders. J 
Gerontol 1994;49:M140-7.

8. Vellas BJ, Wayne SJ, Romero LJ, Baumgartner RN, Garry PJ. Fear of falling and restriction of mobility in elderly fallers. Age Ageing 1997;26:189-93.

9. Arfken CL, Lach HW, Birge SJ, Miller JP. The prevalence and correlates of fear of falling in elderly persons living in the community. Am J Public Health 1994;84:565-70.

10. Fletcher PC, Hirdes JP. Restriction in activity associated with fear of falling among community-based seniors using home care services. Age Ageing 2004;33: 273-9.

11. Friedman SM, Munoz B, West SK, Rubin GS, Fried LP. Falls and fear of falling: which comes first? A longitudinal prediction model suggests strategies for primary and secondary prevention. J Am Geriatr Soc 2002;50:1329-35.

12. Cumming RG, Salkeld G, Thomas M, Szonyi G. Prospective study of the impact of fear of falling on activities of daily living, SF-36 scores, and nursing home admission. J Gerontol A Biol Sci Med Sci 2000;55:M299-305.

13. Delbaere K, Crombez G, Vanderstraeten G, Willems T, Cambier D. Fear-related avoidance of activities, falls and physical frailty: a prospective community-based cohort study. Age Ageing 2004;33:368-73.

14. Yardley L, Smith H. A prospective study of the relationship between feared consequences of falling and avoidance of activity in community-living older people. Gerontologist 2002;42:17-23.

15. Maki BE. Gait changes in older adults: predictors of falls or indicators of fear. J Am Geriatr Soc 1997;45: 313-20.

16. Tinetti ME, Richman D, Powell L. Falls efficacy as a measure of fear of falling. J Gerontol 1990;45:P239-43.

17. Yardley L, Beyer N, Hauer K, Kempen G, Piot-Ziegler C, Todd C. Development and initial validation of the Falls Efficacy Scale-International (FES-I). Age Ageing 2005;34:614-9.

18. Delbaere K, Close JC, Mikolaizak AS, Sachdev PS, Brodaty H, Lord SR. The Falls Efficacy Scale International (FES-I): a comprehensive longitudinal validation study. Age Ageing 2010;39:210-6.

19. Kempen GI, Todd CJ, Van Haastregt JC, Zijlstra GA, Beyer N, Freiberger E, et al. Cross-cultural validation of the Falls Efficacy Scale International (FES-I) in older people: results from Germany, the Netherlands and the
UK were satisfactory. Disabil Rehabil 2007;29:155-62.

20. Ruggiero C, Mariani T, Gugliotta R, Gasperini B, Patacchini F, Nguyen HN, et al. Validation of the Italian version of the falls efficacy scale international (FES-I) and the short FES-I in community-dwelling older persons. Arch Gerontol Geriatr 2009;49 Suppl 1:211-9.

21. Park G, Cho B, Kwon IS, Park BJ, Kim T, Cho KY, et al. Reliability and validity of Korean version of Falls Efficacy Scale-International (KFES-I). J Korean Acad Rehabil Med 2010;34:554-9.

22. Tideiksaar R. Geriatric falls: assessing the cause, preventing recurrence. Geriatrics 1989;44:57-61, 64.

23. Song KY, Moon JS, Kang SS, Choi JH. The survey of activities and fear of falling in the community dwelling elderly. J Korea Community Health Nurs Acad Soc 2001;15:324-33.

24. Park YH. Fall risk assessment in the community-dwelling elderly. J Korean Gerontol Nurs 2004;6:170-8.

25. Kim DJ, Kong GM, Moon SH, Suh BH, Lee SW, Kim $\mathrm{SH}$. Risk factors of falls for home staying elderly people in a rural community. J Korean Orthop Res Soc 2008;11:31-6.

26. Yoo IY, Lee JA. Characteristics and factors associated with falls of the community-dwelling elderly in small cities. J Korean Soc Living Environ Syst 2009;16:428-35.

27. Jang IS, Kim DJ. Home safety assessment for fall prevention in elderly people in a rural community. J Korean Gerontol Nurs 2002;4:176-86.

28. Lee JH, Ahn EM, Kim GE, Jeong YK, Kim JH, Lee GA, et al. The effects of falling and fear of falling on daily activity levels in the elderly residing in Korean rural community. J Korean Geriatr Soc 2009;13:79-88.

29. Rubenstein LZ, Josephson KR, Robbins AS. Falls in the nursing home. Ann Intern Med 1994;121:442-51.

30. Lachman ME, Howland J, Tennstedt S, Jette A, Assmann S, Peterson EW. Fear of falling and activity restriction: the survey of activities and fear of falling in the elderly (SAFE). J Gerontol B Psychol Sci Soc Sci 1998;53:P43-50.

31. Li F, Fisher KJ, Harmer P, McAuley E, Wilson NL. Fear of falling in elderly persons: association with falls, functional ability, and quality of life. J Gerontol B Psychol Sci Soc Sci 2003;58:P283-90.

32. Kim SW, Yang YJ, Eo KS, Cho HJ, Kim YS. Fear of falling in elderly persons living in a home for aged. J Korean Acad Family Med 1998;19:1400-9. 
33. Won CW, Rho YG, SunWoo D, Lee YS. The validity and reliability of Korean Instrumental Activities of Daily Living (K-IADL) scale. J Korean Geriatr Soc 2002;6:273-80.

34. Murphy SL, Williams CS, Gill TM. Characteristics associated with fear of falling and activity restriction in community-living older persons. J Am Geriatr Soc 2002;50:516-20.

35. Kempen GI, van Haastregt JC, McKee KJ, Delbaere K, Zijlstra GA. Socio-demographic, health-related and psychosocial correlates of fear of falling and avoid- ance of activity in community-living older persons who avoid activity due to fear of falling. BMC Public Health 2009;9:170.

36. Scheffer AC, Schuurmans MJ, van Dijk N, van der Hooft T, de Rooij SE. Fear of falling: measurement strategy, prevalence, risk factors and consequences among older persons. Age Ageing 2008;37:19-24.

37. Boyd R, Stevens JA. Falls and fear of falling: burden, beliefs and behaviours. Age Ageing 2009;38:423-8. 